\title{
ELECTRON CLOUD AND BEAM SCRUBBING IN THE LHC
}

\author{
O. Brüning, F. Caspers, I.R. Collins, O. Gröbner, B. Henrist, N. Hilleret*,
} J.-M. Laurent, M. Morvillo, M. Pivi, F. Ruggiero, X. Zhang, CERN, Geneva, Switzerland

\begin{abstract}
An adequate dose of photoelectrons, accelerated by lowintensity proton bunches and hitting the LHC beam screen wall, will substantially reduce secondary emission and avoid the fast build-up of an electron cloud for the nominal LHC beam. The conditioning period of the liner surface can be considerably shortened thanks to secondary electrons, provided heat load and beam stability can be kept under control; for example this may be possible using a special proton beam, including satellite bunches with an intensity of $15-20 \%$ of the nominal bunch intensity and a spacing of one or two RF wavelengths. Based on recent measurements of secondary electron emission, on multipacting tests and simulation results, we discuss possible 'beam scrubbing' scenarios in the LHC and present an update of electron cloud effects.
\end{abstract}

\section{INTRODUCTION AND SUMMARY}

An effective solution to reduce the heat load due to electron cloud build-up in the LHC dipoles is a beam screen with ribbed surface ${ }^{1}$ and reduced reflectivity [1], provided the maximum secondary electron yield $\delta_{\max }$ can be kept below a critical value that for nominal LHC parameters is about 1.3 . For example a $10 \%$ reflectivity gives an acceptable heat load of about $200 \mathrm{~mW} / \mathrm{m}$ for $\delta_{\max }=1.2$, assuming a photoelectron yield $\delta_{\gamma \mathrm{e}} \simeq 0.2$ and a characteristic energy of $5 \mathrm{eV}$ for the secondary electrons. However for a maximum secondary yield $\delta_{\max }=1.8$, i.e. above the critical value, the heat load remains around $5 \mathrm{~W} / \mathrm{m}$ inspite of the lower reflectivity.

Secondary emission can be reduced by special coatings or by an adequate electron dose. As discussed in the following two sections, an electron dose of $1 \mathrm{mC} / \mathrm{mm}^{2}$ is sufficient to lower the maximum secondary yield below the critical value of 1.3. Therefore 'beam scrubbing' scenarios are under study to condition the liner surface in the shortest possible time, while keeping the heat deposition within acceptable bounds. For example the nominal bunch intensity of $10^{11}$ protons can be reduced by a factor 4 or the nominal bunch spacing of $25 \mathrm{~ns}$ can be doubled; in both cases the heat load for $\delta_{\max }=1.8$ becomes about $400 \mathrm{~mW} / \mathrm{m}$ at $7 \mathrm{TeV}$ and can be further reduced by stopping the ramp at an intermediate energy. Another solution

\footnotetext{
*Email: Noel.Hilleret@cern.ch

${ }^{1}$ The corresponding low-frequency inductive impedance, estimated by M. D'Yachkov to a few $\mathrm{m} \Omega$, is very small compared to the LHC impedance budget of some $250 \mathrm{~m} \Omega$. The high frequency behaviour of the impedance and the parasitic losses for a periodic ribbed (or slotted) surface, especially in connection with a possible surface wave, are being investigated by A. Mostacci; according to preliminary estimates they should be negligible.
}

to increase the critical yield during the conditioning period, possibly more effective in terms of beam stability control, is to have satellite bunches with an intensity of $15-20 \%$ of the nominal bunch intensity and a spacing of $5 \mathrm{~ns}$ (two RF wavelengths). Such satellites behave as clearing bunches and remove slow secondary electrons before the next nominal bunch arrives; for reduced reflectivity the corresponding critical yield can be increased to almost a value of two.

To get a rough estimate of the minimum time required for surface conditioning ${ }^{2}$, let us assume a maximum heat load of $200 \mathrm{~mW} / \mathrm{m}$, compatible with cooling, and an average electron energy around $200 \mathrm{eV}$. This is consistent with simulation results discussed in Section 4 for a nominal LHC proton beam with satellite bunches. The corresponding linear flux of electrons bombarding the screen surface is $6 \times 10^{15} \mathrm{~s}^{-1} \mathrm{~m}^{-1}$. Since a meter of LHC beam screen has a surface of $1.25 \times 10^{5} \mathrm{~mm}^{2}$, the electron dose accumulated per hour is $\frac{200 \mathrm{~mW} / \mathrm{m}}{200 \mathrm{eV}} \frac{\mathrm{m}}{1.25 \times 10^{5} \mathrm{~mm}^{2}} 1.6 \times 10^{-19} \mathrm{C} \simeq$ $8 \times 10^{-9} \frac{C}{\mathrm{~mm}^{2} \mathrm{~s}}$ and the beam scrubbing time required to accumulate the required electron dose of $1 \mathrm{mC} / \mathrm{mm}^{2}$ is about 35 hours.

\section{MEASUREMENTS OF SECONDARY ELECTRON YIELD}

The secondary electron yield $\delta_{\mathrm{SEY}}$ of metals is depending drastically on the composition and the roughness of the surface. It is therefore very important to measure the real $\delta_{\mathrm{SEY}}$ of technical materials used in accelerators such as the copper colaminated on stainless steel, the proposed material for the LHC beam screen. The $\delta_{\mathrm{SEY}}$ of a copper surface can be modified by surface treatments like titanium nitride deposition [3], air oxidation [4] or by in situ electron bombardment. This latter effect was first reported by M. Lavarec et al in Ref. [5]. Further investigation carried out at CERN have shown that this effect also exists for stainless steel, aluminium and copper. Figure1 shows the variation of $\delta_{\mathrm{SEY}}$ as a function of the primary electron energy, for a sample of copper colaminated on stainless steel, before and after electron bombardment. This bombardment was made in an unbaked vacuum system at a pressure of $10^{-5} \mathrm{~Pa}$, using $500 \mathrm{eV}$ electrons and corresponded to an electron dose of $5 \times 10^{-3} \mathrm{C} / \mathrm{mm}^{2}$. The maximum yield $\delta_{\max }$ decreased from 2.2 at an electron energy of $300 \mathrm{eV}$ in the initial stage to 1.2 for an electron energy of $450 \mathrm{eV}$ after this bombardment. The variation of $\delta_{\mathrm{SEY}}$ during the bombardment under similar experimental conditions is shown in Fig. 2. The yield measured at the bombardment energy (respectively

\footnotetext{
${ }^{2}$ This estimate, independent of reflectivity and photoelectron yield, has been suggested by $\mathrm{C}$. Benvenuti.
} 
$500 \mathrm{eV}$ and $100 \mathrm{eV}$ ) is plotted as a function of the dose received by the sample. Below a dose of $10^{-6} \mathrm{C} / \mathrm{mm}^{2}, \delta_{\mathrm{SEY}}$ does not change significantly and correponds to the 'true yield' of the surface. For higher doses it decreases towards a stable value reached for a dose greater than $10^{-3} \mathrm{C} / \mathrm{mm}^{2}$. The effect is similar for both primary energies of $100 \mathrm{eV}$ and $500 \mathrm{eV}$. Although not fully understood, this effect can explain, at least partly, the efficiency of the well known procedure of 'RF conditioning' in RF devices. Other experiments have shown that the alteration of the yield is localised to the electron impact region and is permanent under vacuum. Part of the $\delta_{\mathrm{SEY}}$ reduction remains after an air exposure and the colour of the copper surface is slightly changed at the location of the beam impact. More investigations are underway to elucidate the origin of this very useful effect.

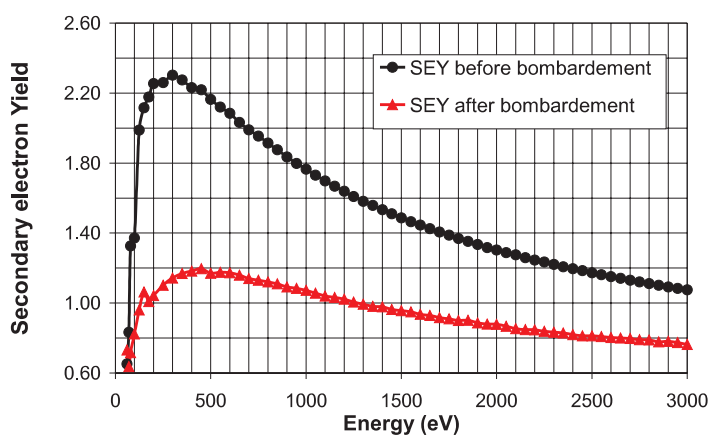

Figure 1: Variation of $\delta_{\mathrm{SEY}}$ as a function of the primary electron energy, for a sample of copper colaminated on stainless steel, before and after bombardment with $500 \mathrm{eV}$ electrons, corresponding to a dose of $5 \times 10^{-3} \mathrm{C} / \mathrm{mm}^{2}$.

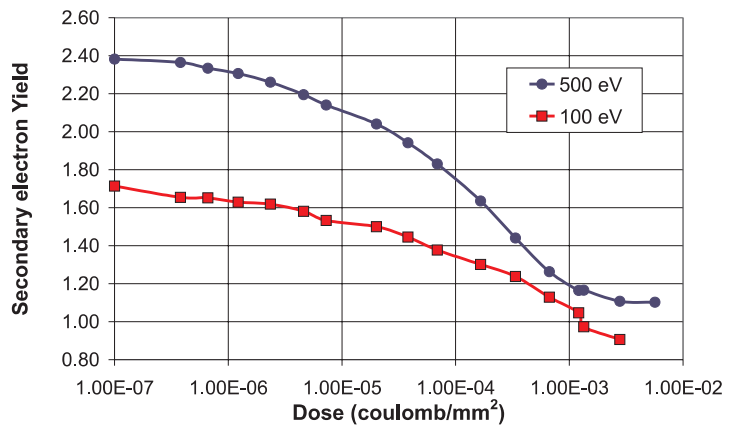

Figure 2: Secondary electron yield measured at the bombardment energy (respectively $500 \mathrm{eV}$ and $100 \mathrm{eV}$ ) as a function of the dose received by the sample.

\section{MULTIPACTING TESTS}

We have investigated beam-induced multipacting by means of a travelling-wave coaxial multi-wire chamber, the electric field produced by a bunched proton beam being simulated by short square RF pulses applied to six equispaced wires parallel to the axis of a $1.4 \mathrm{~m}$ long stainless steel vacuum chamber with $100 \mathrm{~mm}$ diameter. The output from the amplifier, driven by a pulse generator, is DC free and a bias voltage has to be applied to the wires to shift the pulses by the desired voltage; the power coming out from the chamber is then absorbed by a line load. Electrons close to the chamber wall are accelerated towards the center of the chamber by the pulsed electric field. They may reach the opposite side of the chamber and produce secondary electrons if their energy is sufficient. Resonance conditions are met if the next pulse is present at that time, and as a result, the electron multiplication grows up exponentially. Multipacting build-up is recorded by a positively biased electron pick-up, consisting of a round button probe with $1 \mathrm{~cm}$ diameter. Evidence of multipacting instability in the chamber is given by a fast pressure increase, while a negative current is recorded at the pick-up. In addition a complete suppression of the electron multiplication may be obtained by applying a solenoidal magnetic field with an intensity of only a few gauss. For a fixed pulse amplitude of $140 \mathrm{~V}$ and a period of $20 \mathrm{~ns}$, multipacting is observed in a window of pulse widths between 7 and $16 \mathrm{~ns}$. A similar behaviour is measured for the same pulse amplitude and a fixed width of $10 \mathrm{~ns}$, in a window of pulse periods between 17 and $22 \mathrm{~ns}$.

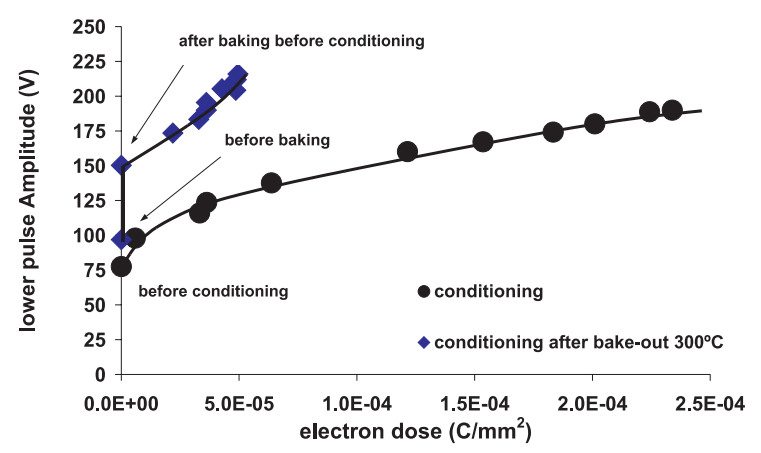

Figure 3: Minimum pulse amplitude required for multipacting as a function of the integrated electron dose: before bake-out (lower curve) or after bake-out at $300^{\circ}$.

Consistently with the results discussed in the previous section, a multipacting intensity decreasing exponentially with time has been monitored by measuring the minimum pulse amplitude needed to trigger the electron multiplication (see Fig. 3). Surface conditioning due to electron bombardment results in a reduction of the secondary emission yield, and the pulse amplitude has to be increased to supply the electrons with sufficient energy to have an average $\delta_{\mathrm{SEY}}>1$ at the wall. After baking the cavity, the minimum pulse amplitude required for multipacting is increased by $50 \%$. In addition, the same cleaning effect is achieved with one order of magnitude less electron dose. The latter is estimated by integrating the current measured by the electron pick-up during multipacting and normalising the accumulated electric charge by the pick-up surface.

An energy spectrum analyzer has been used to measure the energy distribution of the electrons hitting the wall of the chamber during multipacting. Such distribution is peaked around a single energy value and has a typical width of $10 \mathrm{eV}$. Figure 4 shows the linear dependence of the en- 
ergy peak from 40 to $85 \mathrm{eV}$ on the pulse amplitude from 80 to $200 \mathrm{~V}$, then for higher electric fields the electrons are slightly decelerated before they reach the opposite side of the chamber, due to the electric field configuration.

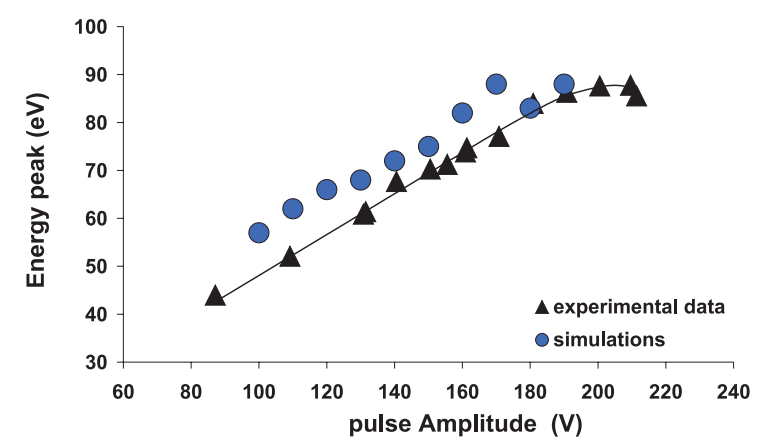

Figure 4: Peak of the energy distribution for the electrons hitting the wall during multipacting as a function of the pulse amplitude: experimental data (triangles) are in relatively good agreement with simulation results (circles).

\section{SIMULATION RESULTS}

The simulation results shown in Fig. 5 refer to the LHC dipole beam screen and have been performed assuming a photoelectron yield $\delta_{\gamma \mathrm{e}} \simeq 0.2$ and a surface reflectivity of $10 \%{ }^{3}$. The maximum secondary electron yield corresponds to a primary electron energy of $300 \mathrm{eV}$ and secondary electrons have a Gaussian energy distribution with $5 \mathrm{eV}$ r.m.s. value and cut-off at 5 sigma. There are 50 slices per bunch and again 50 slices for each inter-bunch gap.

With nominal LHC bunch intensity and spacing, but with satellite bunches at a distance of 2 RF buckets, the heat load for $\delta_{\max }=1.8$ is $180 \mathrm{~mW} / \mathrm{m}$ and the estimated scrubbing time is 43 hours. As shown in Fig. 6 there is a window around $15-20 \%$ for the relative intensity of satellite bunches, where the heat load is significantly reduced; the corresponding critical value of $\delta_{\max }$ is large (above 1.8). This effect is less pronounced for satellites at a distance of only one RF wavelength. For lower intensities of the satellite bunches, the effect of space charge repulsion is reduced and the heat load increases. For a reduced reflectivity of $2 \%$ and a photoelectron yield of 0.1 , the heat load becomes only $15 \mathrm{~mW} / \mathrm{m}$ and the corresponding scrubbing time increases to about 45 days. This is the same time estimated by taking into account only photoelectrons.

\section{REFERENCES}

[1] V. Baglin et. al., "Beam-induced electron cloud in the LHC and possible remedies", CERN LHC Project Report 187 (1998) and Proc. EPAC'98, Stockholm (1998).

[2] M. Furman and G.R. Lambertson, 'The Electron-Cloud Instability in the Arcs of the PEP-II Positron Ring', LBNL41123/CBP Note-246/PEP-II AP Note AP 97.27 (1998),

\footnotetext{
${ }^{3}$ This means that $10 \%$ of the photoelectrons are uniformly distributed around the beam screen, while the remaining $90 \%$ have a Gaussian angular distribution with an r.m.s. angle of $22.5^{\circ}$ from the horizontal plane.
}

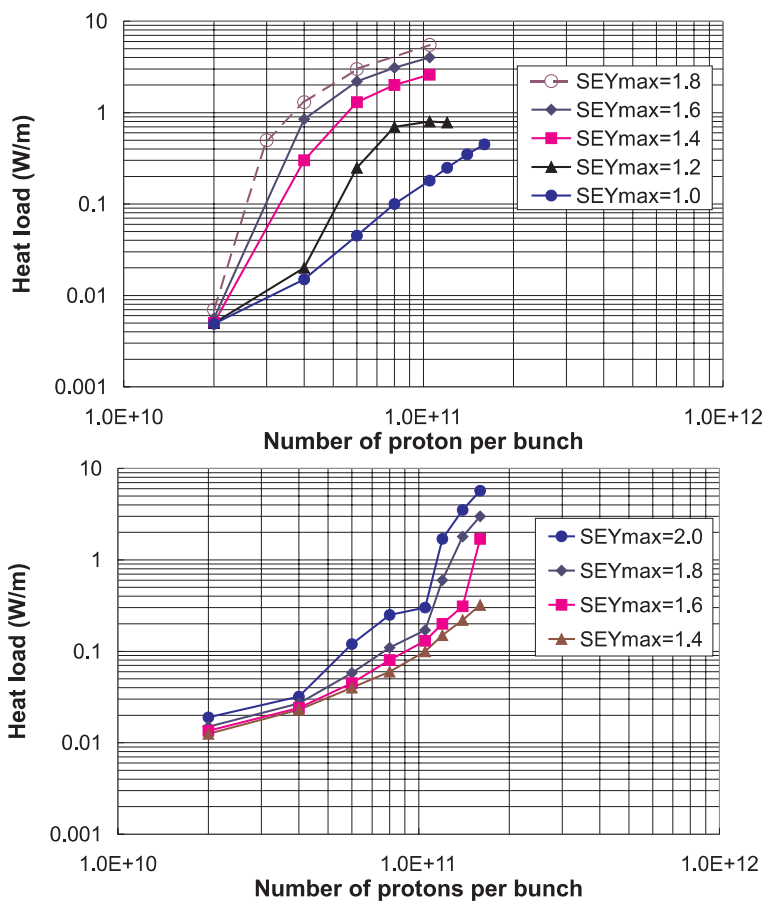

Figure 5: Heat load versus bunch population for different values of $\delta_{\max }$ and $10 \%$ reflectivity: without (top) and with (bottom) satellite bunches having $20 \%$ of the nominal bunch intensity and a spacing of $5 \mathrm{~ns}$.

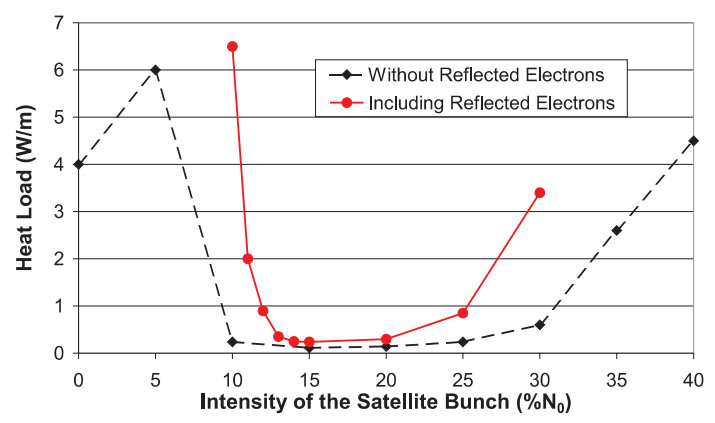

Figure 6: Heat load vs relative intensity of satellite bunches, following nominal LHC bunches at 2 RF wavelengths, with (solid line) or without (dashed line) elastic electron reflection as described in Ref. [2], with $\delta_{\max }=1.6$ and $10 \%$ reflectivity.

Proc. MBI'97, KEK, Tsukuba (1997). See in particular Eqs. (4.10) and (4.11).

[3] E.L. Garwin, F.K. King, R.E. Kirby, "Surface properties of metal-nitride and metal-carbide films deposited on $\mathrm{Nb}$ for radio-frequency superconductivity", J. Appl. Phys. 61, 11451153, (1987).

[4] C. Scheuerlein, I. Bojko, J.L. Dorier, N. Hilleret, "Der Einfluss von Luft auf die Sekundäreelektronenrate von Kupfer" to be published in DPG-Frühjahrstagung des AK Festkörperphysik Münster, 22-26 March 1999.

[5] M. Lavarec, G. Boquet and A. Septier, "The lowering of the secondary electron emission coefficient of real surfaces due to the primary electron bombardment", Proc. 8th Int. Symposium on Discharges and Electrical Insulation in Vacuum, Albuquerque (1978). 